\title{
Preliminary safety estimate of cosmetic anti-age creams with chokeberry extract, using in vivo bioengineering techniques
}

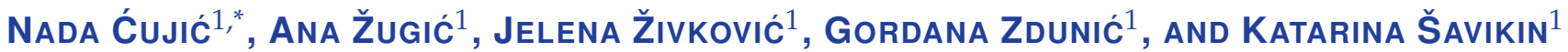 \\ ${ }^{1}$ Institute for Medicinal Plants Research "Dr. Josif Pančić", Belgrade, Serbia \\ *Corresponding author: ncujic@mocbilja.rs
}

Received: November 27, 2017

Accepted: December 15, 2017

Published on-line: December 16, 2017

Published: December 25, 2017

\begin{abstract}
Potential of anti-age cosmetic line, day and night creams were evaluated using noninvasive measurements of the appropriate skin biophysical parameters in a 24-h in vivo study under occlusion. Thirtyone healthy female volunteers were involved in the study. Electrical capacitance (EC), transepidermal water loss (TEWL), erythema index (EI) were evaluated. The preliminary safety profile of the investigated creams with natural origin ingredients based on obtained results can be considered satisfactory. The stated samples did not cause the change in the measured biophysical parameters-TEWL and EI, while they increased EC i.e. stratum corneum hydration. These results suggest that chokeberry extract and other natural ingredients could exert beneficial effects and has potential on aged skin thereby slowing down the appearance of wrinkles and their existing alleviation, and they could be considered preferable in the cosmetic products intended for the treatment of aged skin.
\end{abstract}

Key words: chokeberry, polyphenols, anthocyanins, antioxidants, anti-age

http://dx.doi.org/10.5937/leksir1737041C

\section{INTRODUCTION}

In the last years, there is a growing trend of natural cosmetics utilization, which is related with the frequent occurrence of allergies and skin irritations caused by synthetic ingredients commonly included in the formulation of these products (synthetic preservatives-parabens, colors, stabilizers, etc.) (Glampedaki and Dutschk, 2014). Antioxidants, especially naturally derived from plants, has been recognized as an excellent source of bioactive components with beneficial effects on skin, especially to preventing premature skin aging and wrinkling (Shahidi and Ambigaipalan, 2015). There is an increasing research interest to polyphenolic compounds which have proven antioxidant activity and have shown promising effects applied as cosmetic active ingredients (Bräunlich et al., 2013; Shahidi and Ambigaipalan, 2015). Berry fruits, chokeberry, in particular, are one of the richest sources of natural antioxidants as anthocyanins, flavonols, procyanidins and phenolic acids(Bräunlich et al., 2013; Kulling and Rawel, 2008; Rugina et al., 2012; Sueiro et al., 2006). Chokeberry (Aronia melanocarpa [Michx] Elliot, Rosaceae) is a rich source is a perennial shrub native to North America and it was introduced to Eastern Europe, Scandinavia, and Russia in early $20^{\text {th }}$ century (Kokotkiewicz et al., 2010; Kulling and Rawel, 2008).

It is well known that the aging process could modify struc- tural and mechanical properties of the skin through changes of the elastic and collagen fibers (Ryu et al., 2008). As aging is followed by a skin elasticity reduction which begins in the early twenties, anti-age cosmetics could have many beneficial effects on the skin. It could intensely hydrate and nourish the skin and also be useful for skin biomechanical characteristics, could prevent premature skin aging and delay the onset of first and reduce existing wrinkles (Kim et al., 2014). Daily use of anti-age cosmetics could also revitalize the skin, prevent moisture loss, stimulate the formation of new cells and collagen and reduce the harmful effects of external factors (Glampedaki and Dutschk, 2014; Kim et al., 2014).

The aim of this study was to create anti-age formulations which contain natural ingredients of plant origin that have positive effects on the skin aging symptoms reduction and could significantly reduce the use of synthetic ingredients that have been shown side effects.Preliminary safety profile/irritation potential of the developed cosmetic products (active day and night creams-D and $\mathrm{N}$, as well as their matching vehicles (placebo samples)-D-P and N-P, respectively), was evaluated using noninvasive measurements of the appropriate biophysical parameters of the skin in a 24-h in vivo study under occlusion. Non-invasive skin biophysical measurements are widely used in the evaluation of skin conditions. 
Table 1. The effects of day and night anti-age creams on skin biophysical parameters in healthy females

\begin{tabular}{llccc}
\hline Time & Treatment & EC & EI & $\begin{array}{c}\text { TEWL } \\
\text { [g/m2h] }\end{array}$ \\
\hline Baseline & UC & & & $10.19 \pm 0.87 \mathrm{ab}$ \\
& N & $36.61 \pm 2.78 \mathrm{c}$ & $267.14 \pm 15.67$ & $7.85 \pm 0.67 \mathrm{c}$ \\
& N-P & $36.39 \pm 2.77 \mathrm{c}$ & $238.44 \pm 13.99$ & $7.67 \pm 0.65 \mathrm{c}$ \\
& UCO & $35.63 \pm 2.71 \mathrm{c}$ & $248.26 \pm 14.57$ & $9.33 \pm 0.80 \mathrm{a}-\mathrm{c}$ \\
& D & $38.62 \pm 2.94 \mathrm{bc}$ & $264.68 \pm 15.53$ & $8.07 \pm 0.69 \mathrm{bc}$ \\
& D-P & $36.64 \pm 2.79 \mathrm{c}$ & $244.53 \pm 14.35$ & $8.40 \pm 0.72 \mathrm{bc}$ \\
\hline After 24-h & UC & $33.32 \pm 2.53 \mathrm{c}$ & $253.15 \pm 14.85$ & $10.68 \pm 0.91 \mathrm{a}$ \\
& N & $36.16 \pm 2.75 \mathrm{c}$ & $249.61 \pm 14.65$ & $8.50 \pm 0.72 \mathrm{a}-\mathrm{c}$ \\
& N-P & $51.76 \pm 3.94 \mathrm{a}$ & $233.64 \pm 13.71$ & $8.13 \pm 0.69 \mathrm{bc}$ \\
& UCO & $51.85 \pm 3.94 \mathrm{a}$ & $236.47 \pm 13.87$ & $8.84 \pm 0.75 \mathrm{a}-\mathrm{c}$ \\
& D & $39.15 \pm 2.98 \mathrm{a}$ & $258.47 \pm 15.16$ & $8.21 \pm 0.70 \mathrm{bc}$ \\
& D-P & $50.87 \pm 3.87 \mathrm{a}$ & $237.55 \pm 13.94$ & $8.51 \pm 0.73 \mathrm{a}-\mathrm{c}$ \\
\hline
\end{tabular}

a Treatment abbreviations: UC-control without occlusion; UCO-untreated control under occlusion; N-standard night cream; D-standard day cream; N-Pincorporated hydrophobic night cream vehicle; D-P-incorporated hydrophilic day cream vehicle.

${ }^{b}$ Observed parameters: EC-electrical capacitance; TEWL-transepidermal water loss; EI-erythema index.

${ }^{c}$ Values are means \pm standard deviation $(n=31)$. Mean values with different letters are significantly different at $\mathrm{P}<0.05$.

\section{MATERIALS AND METHODS}

\section{Creams preparations}

Chokeberry propylene glycol extract $(3 \%, \mathrm{w} / \mathrm{v}) \quad(1: 3.5$, solid:solvent ratio), with a slight addition of hibiscus extract $(0.5 \%, \mathrm{w} / \mathrm{v})(1: 2.5$, solid:solvent ratio), which were used for creams preparations, were obtained from Production Sector of the Institute for Medicinal Plant Research "Dr. Josif Pančić". Extracts were prepared using $45 \%$ propylene glycol and extraction was done by percolation method. Propylene glycol is normally recommended as a solvent for the preparation of the extracts which could be incorporated in cosmetic products, due to the beneficial effect on the skin. Alcohol was not used as a solvent for the extraction. In addition to the composition of creams also were incorporated the following active components: hyaluronic acid, avocado oil, grape seed oil, shea butter, elastin, vitamin C, vitamin E, elastin, natural wetting factors of the skin. The formulation was made without parabens, paraffin, synthetic dyes and alcohol. Parabens have been replaced by less harmful preservatives. The formulation was free of paraffin and alcohol in order to grease the skin and impair the natural skin barrier. Synthetic dyes have not been used in these preparations, where natural colors originating from the extracts have been used as a substitute. Day and night creams$\mathrm{D}$ and $\mathrm{N}$ were made by a standard method (mixing the oil and aqueous phase) (IKA Combimag $\mathrm{RCH}$, Germany) with a few modifications during the heating, in order to preserve the stability of sensitive active substances (avocado oil, grape seed oil, mixture of extracts, vitamins C and E, UV filter, elastin, hyaluronic acid, natural wetting factors of the skin), which were added at below $40^{\circ} \mathrm{C}$. Placebo samples were prepared without the active substances. $\mathrm{pH}$ values of $\mathrm{D}$ and $\mathrm{N}$ creams corresponded to a $\mathrm{pH}$ value of the skin and therefore were eligible for the skin applications.

\section{Protocol of preliminary safety profile/irritation study of creams}

Thirty-one healthy female volunteers (mean age $46.1 \pm 6.6$ ), which participated in the study, were thoroughly informed about the possible treatment effects and the protocol of the examination prior to signing written consents, in accordance with the Helsinki Declaration. The study was approved by the Ethical Committee of the Institute for Medicinal Plant Research "Dr. Josif Pančić", Belgrade, Serbia (Decision No 01-9337-13). Healthy females without the clinical signs of dermatological disease participated in the study. The following parameters were evaluated: electrical capacitance (EC) (quantifying the stratum corneum hydration), erythema index (EI) as a measure of skin color and transepidermal water loss (TEWL) as a measure of skin barrier function, using Multi Probe Adapter MPA®9 (Courage and Khazaka Electronic $\mathrm{GmbH}$, Germany). Corneometer ${ }^{\circledR C M} 825$ was used for skin measurement of EC, TEWL was measured using Tewameter®TM210 and EI using Mexameter ${ }^{\circledR M X 18, ~ r e s p e c t i v e l y . ~ A l l ~}$ measurements were conducted on flexor aspects of forearms at square application sites of $9 \mathrm{~cm}^{2}(3 \times 3 \mathrm{~cm})$, leaving a site per arm as a rectangle next to the wrist as an untreated control (UC) on the right forearm and as untreated control occluded (UCO) on the left forearm. Treatments included in this experiment were: $\mathrm{N}$ - night cream; D-day cream; N-P-night cream matching vehicle-placebo; D-P-day cream matching vehicle-placebo. The volunteers were instructed not to use dermopharmaceutical and/or cosmetic products on the tested areas a three days before and throughout the study, but were allowed to wash skin normally before the participation in the study and as well as to spend at least 30 minutes in a room in which the measurements were conducted in order to adapt to the temperature and relative humidity. After initial measurements (baseline), $0.016 \mathrm{~g} / \mathrm{cm}^{2}$ of each sample cream were applied, subsequently coated with immediately covered with Parafilm ${ }^{\circledR}$ (Pechiney Plastic Packaging, Inc., Menasha, Wisconsin, USA) and fixed with hypoallergenic cotton adhesive tape Sensifix ${ }^{\circledR}($ Belgrade, Serbia). Two hours after occlusion removal of the 24-h occlusion, all parameters were reassessed (Jaksic et al., 2012; Tasic-Kostov et al., 2011).

\section{Statistical analysis}

Significant differences between all examined parameters (different skin areas for the same measured parameters, at baseline and after 24-h occlusion) were analyzed by one-way ANOVA. Tukey's Significant Difference test was used as the post hoc test, and the means were considered significantly different at level $\mathrm{P}<0.05$. All measurements were done in triplicate and statistical analyses were performed using Microsoft Office Excel 2010.

\section{RESULTS AND DISCUSSION}

For the purpose of this research, anti-age cosmetic line consisting of day (D) and night (N) creams was developed, 


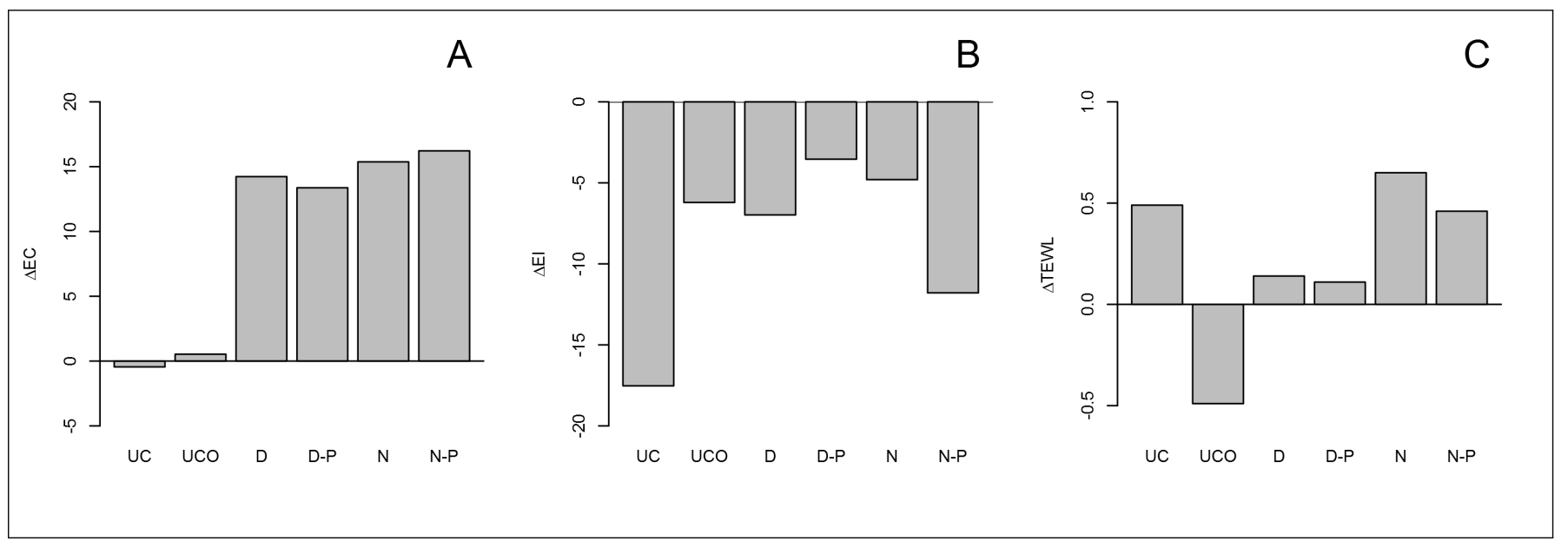

Fig. 1. Differences between baseline values vs. values after 24-h occlusion for all measured parameters and tested areas; A - electrical capacitance (EC); B - erythema index (EI); C - transepidermal water loss (TEWL); Treatment abbreviations: UC - control without occlusion; UCO untreated control under occlusion; N - standard night cream; D - standard day cream; N-P - night cream matching vehicle-placebo; D-P - day cream matching vehicle-placebo.

both based on natural raw materials. In these preparations were incorporated new active substances that have been rarely used in cosmetics and also the components that have already well-known effect on the skin. In the stated creams (D and N), hyaluronic acid, as well as black chokeberry and hibiscus propylene glycol extracts were incorporated as active ingredients in the appropriate hydrophilic (day cream) i.e. hydrophobic (night cream) vehicles. To the best of our knowledge, literature data about incorporated chokeberry extract in creams have not been previously published. For the products of pharmaceutical, cosmetic and food industry, quality of the extracts as components of the products is critical and increment of the amount of biologically active phenolics in extracts is a challenging task.

All the female volunteers completed the study and reported strict compliance with the given instructions. Noninvasive skin biophysical measurements were used in the evaluation of skin conditions. It was monitored hydration of the skin (EC), the most valid measurement for assessment of low irritant skin reactions (EI), as well as TEWL measurement, which is a highly sensitive and precise method for the determination of skin water loss (Table 1). It is known that occlusion itself could induce barrier damage and may cause irritation (Zhai and Maibach, 2002). In order to eliminate the influence of occlusion itself, final measurements of the monitored parameters were performed $2 \mathrm{~h}$ after occlusion removal, when the effects of occlusion have been finished.

Two hours after occlusion removal, all the investigated samples led to significant improvement of EC compared to baselines and controls, revealing skin hydration potential probably related to appropriate vehicles themselves (D-P and $\mathrm{N}-\mathrm{P}$ ), bearing in mind lack of significant differences after treatment with these samples compared to the matching active creams ( $D$ and $N$, respectively). Since active and placebo samples are based on the same carrier system, the obtained results might indicate that for skin hydration, the carrier itself is beneficial. In order to investigate the irritation potential of creams, EI was monitored. There was no significant change in EI, indicating well-tolerated skin formulations. The investigated samples showed overall satisfying preliminary safety profiles (low in vivo irritation potential). The most common method of evaluation of the skin barrier function, TEWL was also conducted. There was no significant increase of TEWL for the investigated samples compared to the baseline values. Significant improvement of this parameter was detected for the sample N and UC, but this increase cannot be attributed to the treatment with this sample itself, while the same difference between test-spots $\mathrm{N}$ and UC was also noted in the initial measurement (Table 1). The in vivo measured parameters (EC, EI and TEWL) were expressed as absolute changes ( $\Delta$ values) compared to the baseline (Figure 1). After treatment, significant increase of $\mathrm{D}$, $\mathrm{D}-\mathrm{P}, \mathrm{N}$ and N-P in EC have been seen when compared to the $\mathrm{UC}$ and UCO values (Figure 1A). Although occlusion can significantly increase skin hydration by blocking water loss from the surface, the obtained results cannot be attributed to the occlusion then to the effects of the investigated active principles. After application, EI, as an indicator of the skin irritation by the measurement of skin redness and inflammation, was not significantly changed compared to the baseline values for any investigated cream sample (Figure 1B).

As already has been said, there were no significant differences in transepidermal water loss measured as TEWL for the investigated samples, except UCO (Figure 1C). This is in accordance with previously published results where also there were not significant changes in TEWL, a marker of skin barrier function (Buraczewska-Norin, 2012; Zhai and Maibach, 2002). The active ingredients of chokeberry extract exhibited strong antioxidant activity and slowing down the appearance of wrinkles and existing wrinkles alleviation, improving microcirculation and strengthens blood vessels. The results of published study (Kim et al., 2014) showed that the anti-wrinkle activities of the chokeberry extract, related to the production of matrix metalloproteinases and collagenase MMP-1, or the production of collagen, were highly correlated with antioxidant activity due to high amounts of anthocyanins in the extract. Beside chokebery extract, day and night creams contained small amount of hibiscus extract, rich with vitamin $\mathrm{C}$ who stimulates the collagen synthesis, a protein which is responsible for skin tonus and elasticity Aburjai and Natsheh (2003). Fruit acids from hibiscus extract effectively stimulate, feed and revitalize the skin. Beside chokeberry polyphenols as naturally powerful antioxidants, creams contained vitamin E which could prevent premature skin aging and wrinkling. Avocado oil, grape seed oil and shea butter effectively feed and revitalize the skin due to the complex of active ingredients which they contain, especially high content of unsaturated fatty acids and phytosterols and they are widely used in the cosmetics industry as moisture agents, which have been 
shown through increasing of EC. In long-term daily use, moisturizers may either improve skin barrier function, and consequently increase penetration of active ingredients, in this case polyphenols (Filipović et al., 2016). Hyaluronic acid, a natural component of the skin located in the extracellular matrix, probably recovered lost skin moisture and gives the visual effect of skin lifting (Scott and Banga, 2015). Creams contained components of natural wetting factors of skin and elastin. Elastin as a protein is responsible for skin tonus, probably gave it back skin moisturize, elasticity and freshness. Creams also contained a complex of UV filters that protect the skin from harmful sun rays. The absence of skin erythema in a 24-h occlusion study, which was seen with no changes in EI, may preliminary indicate the adequate safety profile of the day and night creams and the incorporated cosmetic actives. The achieved hydration effect of the skin is probably attributed to ingredients with water binding capacity (hyaluronic acid, elastin, natural wetting factor) or to physiological based lipids (avocado oil, grape seed oil and shea butter).

\section{CONCLUSION}

The aim of the study was in vivo evaluation of the skin irritation potential and the efficiency of anti-age cosmetic line, with incorporated chokeberry extract and others natural moisturizing ingredients. Using the noninvasive skin biophysical measurements, skin hydration, transepidermal water loss and erythema index were measured on 31 healthy women volunteers. After 24-hour occlusion, there were no significant changes in the erythema index for both tested creams, indicating well-tolerated skin formulations. Two hours after occlusion, all the investigated samples led to significant increasing of skin hydration compared to baselines and controls. Due to the failure of skin irritation and possibility to prevent moisture loss, daily use of $\mathrm{D}$ and $\mathrm{N}$ creams may be safely applied as anti-aging cosmetic line, which could revitalize the skin, reduce the symptoms of skin aging by stimulating the formation of new cells and collagen, and also reduce the harmful effects of external factors, using natural substances which have significantly less side effects then synthetic components. Finally, this study demonstrated that the investigated active substances, especially chokeberry and hibiscus extracts, natural oils, vitamins and hyaluronic acid are effective, indicating that they could be suitable actives for anti-age cosmetics.

\section{ACKNOWLEDGMENTS}

Acknowledgment.This work was supported by the Ministry of Education, Science and Technological Development of the Republic of Serbia, project number 46013.

\section{REFERENCES}

Aburjai, T. and Natsheh, F. M. (2003). Plants used in cosmetics, Phytotherapy research: PTR 17(9): 987-1000.

Bräunlich, M., Slimestad, R., Wangensteen, H., Brede, C., Malterud, K. and Barsett, H. (2013). Extracts, Anthocyanins and Procyanidins from Aronia melanocarpa as Radical Scavengers and Enzyme Inhibitors, Nutrients 5(3): 663-678.

Buraczewska-Norin, I. (2012). Skin Barrier Responses to Moisturizers: Functional and Biochemical Changes, Treatment of Dry Skin Syndrome, Springer, Berlin, Heidelberg, pp. 525544.

Filipović, M., Gledović, A., Lukić, M., Tasić-Kostov, M., Isailović, T., Pantelić, I., Vuleta, G. and Savić, S. (2016).
Alp rose stem cells, olive oil squalene and a natural alkyl polyglucoside emulsifier: Are they appropriate ingredients of skin moisturizers - in vivo efficacy on normal and sodium lauryl sulfate - irritated skin?, Vojnosanitetski Pregled 73: 9911002.

Glampedaki, P. and Dutschk, V. (2014). Stability studies of cosmetic emulsions prepared from natural products such as wine, grape seed oil and mastic resin, Colloids and Surfaces A: Physicochemical and Engineering Aspects 460: 306-311.

Jaksic, I., Lukic, M., Malenovic, A., Reichl, S., Hoffmann, C., Müller-Goymann, C., Daniels, R. and Savic, S. (2012). Compounding of a topical drug with prospective natural surfactant-stabilized pharmaceutical bases: Physicochemical and in vitro/in vivo characterization - A ketoprofen case study, European Journal of Pharmaceutics and Biopharmaceutics 80(1): 164-175.

Kim, S. H., Ahn, J. H., Hong, H. C., Choi, H. Y., Kim, Y. J., Kim, N. H., Yoo, H. J., Kim, H. Y., Seo, J. A., Kim, N. H., Kim, S. G., Choi, K. M., Baik, S. H. and Choi, D. S. (2014). Changes in the clinical manifestations of primary aldosteronism, The Korean Journal of Internal Medicine 29(2): 217.

Kokotkiewicz, A., Jaremicz, Z. and Luczkiewicz, M. (2010). Aronia Plants: A Review of Traditional Use, Biological Activities, and Perspectives for Modern Medicine, Journal of Medicinal Food 13(2): 255-269.

Kulling, S. E. and Rawel, H. M. (2008). Chokeberry (Aronia melanocarpa) - A review on the characteristic components and potential health effects, Planta Medica 74(13): 1625-1634.

Rugina, D., Sconta, Z., Leopold, L., Pintea, A., Bunea, A. and Socaciu, C. (2012). Antioxidant Activities of Chokeberry Extracts and the Cytotoxic Action of Their Anthocyanin Fraction on HeLa Human Cervical Tumor Cells, Journal of Medicinal Food 15(8): 700-706.

Ryu, H. S., Joo, Y. H., Kim, S. O., Park, K. C. and Youn, S. W. (2008). Influence of age and regional differences on skin elasticity as measured by the Cutometer, Skin research and technology 14(3): 354-358.

Scott, J. A. and Banga, A. K. (2015). Cosmetic devices based on active transdermal technologies, Therapeutic Delivery 6(9): 1089-1099.

Shahidi, F. and Ambigaipalan, P. (2015). Phenolics and polyphenolics in foods, beverages and spices: Antioxidant activity and health effects - A review, Journal of Functional Foods 18: 820-897.

Sueiro, L., Yousef, G., Seigler, D., de Mejia, E., Grace, M. and Lila, M. (2006). Chemopreventive Potential of Flavonoid Extracts from Plantation-Bred and Wild Aronia melanocarpa (Black Chokeberry) Fruits, Journal of Food Science 71(8): C480C488.

Tasic-Kostov, M. Z., Reichl, S., Lukic, M. Z., Jaksic, I. N. and Savic, S. D. (2011). Does lactobionic acid affect the colloidal structure and skin moisturizing potential of the alkyl polyglucoside-based emulsion systems?, Die Pharmazie 66(11): 862-870.

Zhai, H. and Maibach, H. I. (2002). Occlusion vs. skin barrier function, Skin research and technology 8(1): 1-6. 